\title{
Rehabilitation of a Patient with Screw-retained Milled Titanium Hybrid Prosthesis in the Mandibular Arch and Immediate Implant Placement in the Region of Maxillary Canine followed by Connective Tissue Graft and Immediate Provisionalization
}

\author{
${ }^{1}$ Nikhil Vasant Jain, ${ }^{2}$ Purva Hemant Shinde, ${ }^{3}$ Affaf Gharatkar, ${ }^{4}$ Gaurav Poplai
}

\begin{abstract}
The continuous resorption of the edentulous mandible is the most common cause for the patients not being satisfied with conventional dentures. With the advent of dental implants, various removable and fixed options can be provided to the patient for better function, retention, esthetics and phonetics. The mandibular implant-supported hybrid prosthesis ia a viable prosthetic option as it fixed for the patient and removable for the clinician. The passive fit of a hybrid prosthesis is a pre-requisite for a successful prognosis, which can be achieved using the newer technology like computer-aided design and computer-aided manufacturing (CAD-CAM) milled titanium frameworks. The use of connective tissue graft to enhance soft tissue bulk around implants helps the clinician provide esthetically appealing outcomes. Immediate loading of the implants is the most preferred option for patients who are esthetically very conscious. Its provisionalization using screw-retained prosthesis ensures a stable peri-implant health as the extruded cement can be eliminated extraorally. This article presents the fabrication of mandibular implant-supported hybrid prosthesis with CAD-CAM milled titanium framework and connective tissue around immediately placed implant with screwretained provisional prosthesis.
\end{abstract}

Keywords: Hybrid prosthesis, Connective tissue graft, Milled titanium framework, PMMA trial, RFA analysis.

How to cite this article: Jain NV, Shinde $\mathrm{PH}$, Gharatkar A, Poplai G. Rehabilitation of a Patient with Screw-retained Milled Titanium Hybrid Prosthesis in the Mandibular Arch and Immediate Implant Placement in the Region of Maxillary Canine followed by Connective Tissue Graft and Immediate Provisionalization. Int J Oral Implantol Clin Res 2014;5(2):83-87.

\footnotetext{
1,2Maxillofacial Prosthodontist and Oral Implantologist

${ }^{3,4}$ Endodontist

1,2Department of Maxillofacial Prosthodontics and Oral Implantology, MA Rangoonwala College of Dental Sciences and Research Center, Pune, Maharashtra, India

${ }^{3,4}$ Department of Endodontics and Conservative Dentistry, MA Rangoonwala College of Dental Sciences and Research Center Pune, Maharashtra, India
}

Corresponding Author: Nikhil Vasant Jain, Maxillofacial Prosthodontist and Oral Implantologist, Department of Maxillofacial Prosthodontics and Oral Implantology, MA Rangoonwala College of Dental Sciences and Research Center, Pune, Maharashtra India, Phone: 02223883890, e-mail: drnikhiljain@outlook.com

\section{Source of support: Nil}

Conflict of interest: None

\section{INTRODUCTION}

Various treatment options are available for edentulous patients who cannot adapt to long-term use of conventional complete dentures. They include implant supported removable (overdentures with locators, bars, magnets, ball attachments) and fixed prosthesis (implant supported fixed dental prosthesis-screw and cement retained, screw retained acrylic and titanium ceramic hybrids).

A hybrid denture is one that is fabricated over a metal framework and retained by screws threaded into the implant abutments. ${ }^{1}$ The advantage of the hybrid prosthesis over the other treatment options is that it is fixed for the patient and removable for the clinician. Maintenance of the restorative structure and implants is needed during the lifetime of such prosthesis, which becomes relatively easier because of the screw-retained nature of the prosthesis. ${ }^{2}$

The metal framework of the hybrid prosthesis requires having a passive fit over the implants to avoid various complications like fracture of the screws and most importantly to avoid torque forces on the implants. One of the most recent approaches to improve passivity of fit is using the laser scanned computer numeric controlled-milled titanium (computer-aided design/computer-aided manufacturing) framework. ${ }^{3}$ With this specific fabrication procedure, the material has high mechanical properties and the inaccuracies of a casted framework are eliminated. ${ }^{4}$

A screw-retained prosthesis avoids the peri-implant complications that occur with the presence of excess luting cement of a cement-retained prosthesis, which is difficult to eliminate completely. Gingival inflammation and progressive bone recession around the implant restorations is a common complication due to the residual cement. ${ }^{5}$

The patient demands for esthetics and sooner completion of their treatment with long-term predictability have increased. A connective tissue graft would be the treatment of choice for achieving adequate peri-implant soft-tissue coverage and bulk over the labial surface of the anterior implants 
for esthetics as well as function and maintenance. ${ }^{6}$ Quicker loading protocols should be adopted for faster completion of the treatment while ensuring high predictability.

The loading protocol is based on the initial implant stability, which can predict its osseointegration and longterm prognosis. Different methods of assessing the implant stability have been used like the resonance frequency analyzer (RFA), which measures the implant stability quotient (ISQ) with the amplitude and magnitude of sound waves that pass through the implant. ${ }^{7}$ This analyzer can be used to assess the implant osseointegration at any stage and also at maintenance phase.

This article presents a mandibular implant screw-retained hybrid prosthesis, maxillary canine implant with connective tissue graft and immediate provisionalization and posterior implants with indirect sinus lift.

\section{CASE HISTORY}

A 55-year-old female patient is referred to the department of prosthodontics with a complaint of missing teeth and wants replacement of the same. She has missing upper left molars

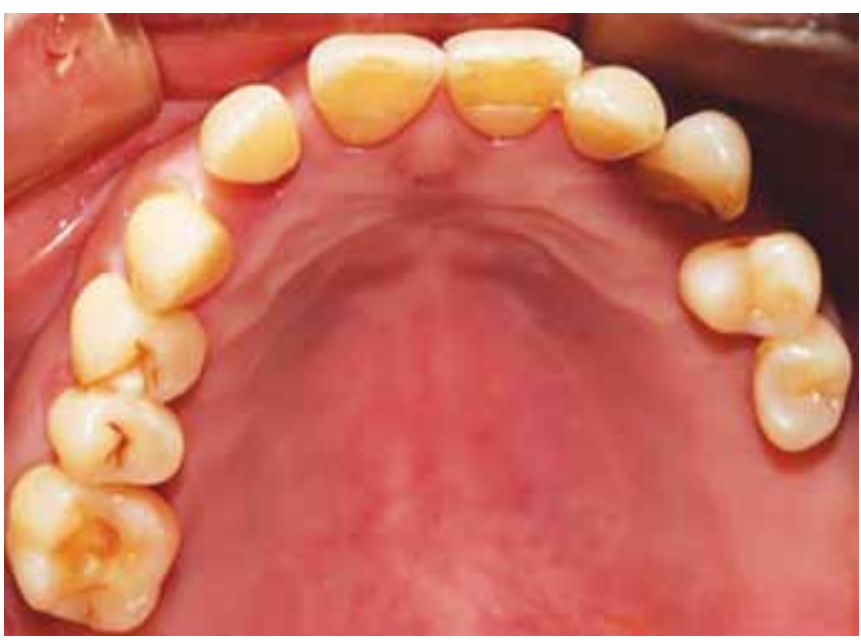

Fig. 1: Maxillary arch showing missing upper left molars

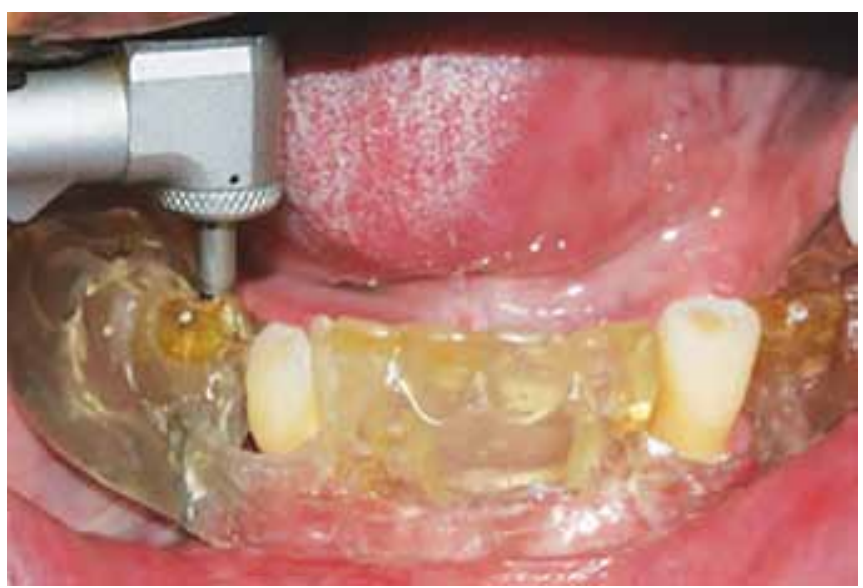

Fig. 3: Stent fabricated for placement of implants at predetermined positions and a periodontally compromised left canine (Fig. 1). The lower arch only has periodontally compromised canines (Fig. 2).

Extraction of the upper left canine is followed by immediate placement of implant with connective tissue graft and immediate provisionalization. As assessed from the cone beam computed tomography (CBCT) scan, indirect sinus lift for the implants in the molar regions was carried out with simultaneous placement. Extraction of the lower canines was followed by placement of six implants in the mandibular arch. An implant-supported hybrid prosthesis is planned on five of these implants with a lone standing posterior implant.

A diagnostic mounting is done on a semi-adjustable articulator following facebow record and jaw relation. A wax trial was carried out keeping in mind the principle of prosthetically driven implant placement. A surgical stent is fabricated using the wax-trial and used to ensure precise placement of the implants (Fig. 3).

\section{IMPLANT SURGERY}

After local anesthetic infiltration, a crestal and a releasing incision were given to raise a full-thickness flap in the upper

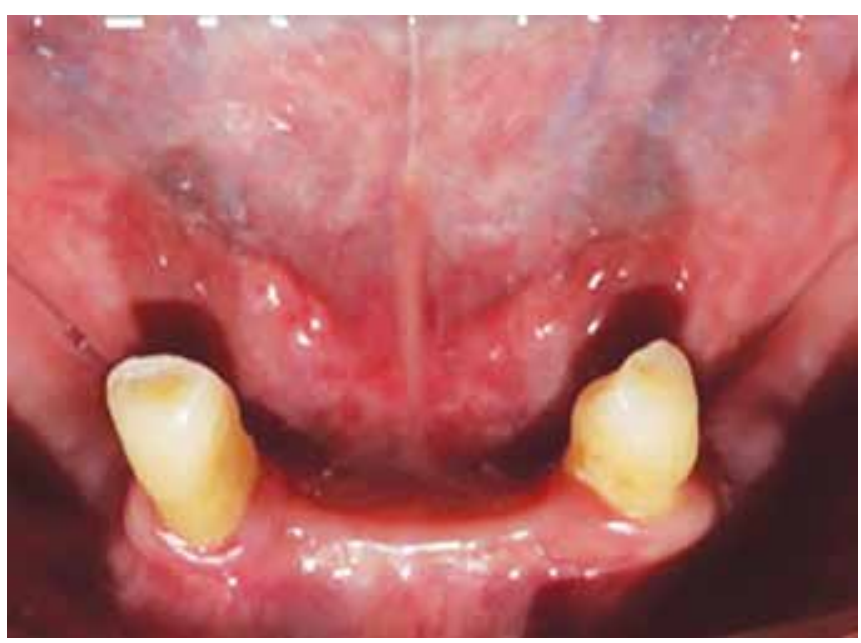

Fig. 2: Mandibular arch showing periodontally compromised canines

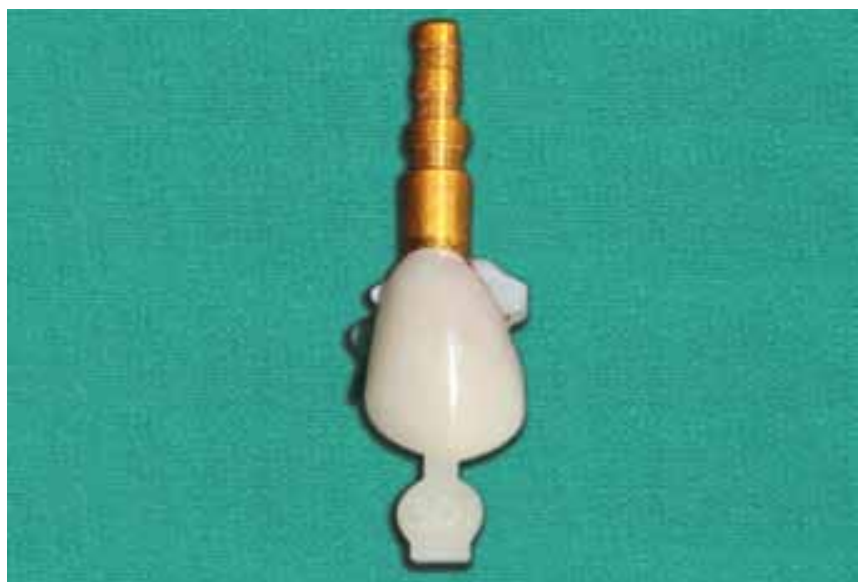

Fig. 4: Polycarbonate crown luted on an abutment attached to an analog 
left molar region and the bone topography was evaluated. Implants (Biohorizons, Birmingham, AL, USA) were placed following indirect sinus lift.

The upper left canine was extracted and immediate implant placement was carried out. The initial stability of the implant was evaluated using the Ostell RFA unit. The ISQ value was 62 , which made it eligible to be temporized immediately. The abutment was attached to the implant and an appropriate size and shape of the polycarbonate crown was selected. The abutment was removed, attached to an analog and the polycarbonate crown luted over it using flowable composite (Fig. 4). The excess cement is removed and it is screw-retained on the implant (Fig. 5). The soft tissue biotype on the labial aspect of the implant was evaluated. Connective tissue graft was harvested from the posterior region of the palate (Fig. 6) and placed on the labial aspect in the region of the canine (Fig. 7).

In the mandibular arch, the surgical stent is used to place implants bilaterally in the regions of lateral incisors, first premolars and first molars. Paralleling pins were used as a guide to orient implants in appropriate position. The flap

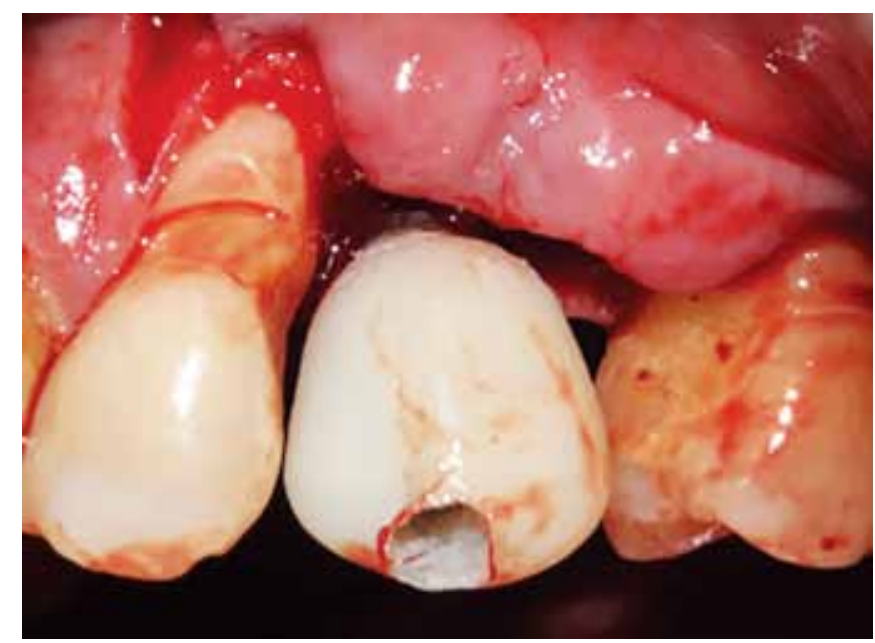

Fig. 5: Screw-retained temporary prosthesis

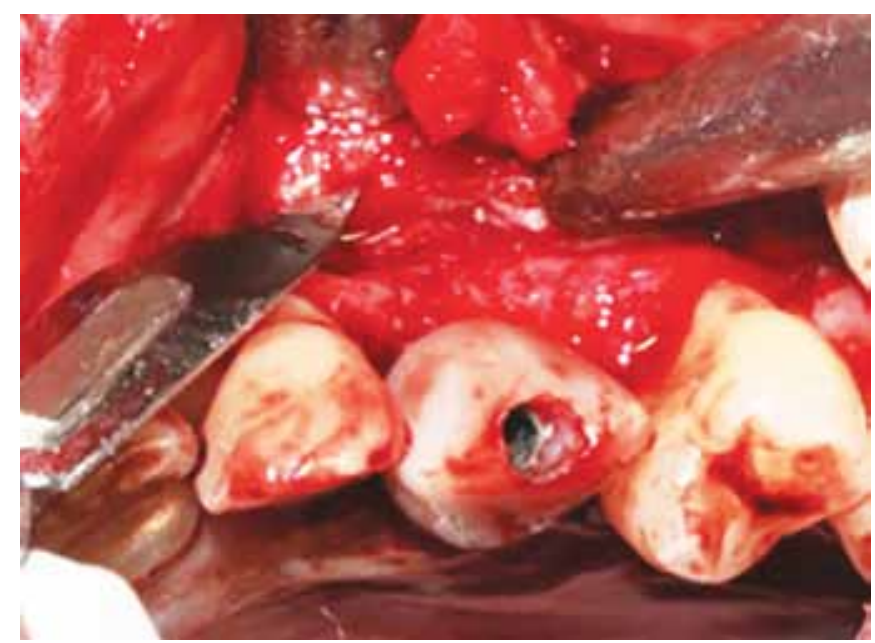

Fig. 7: Connective tissue graft placed in the region of canine was repositioned and tension free sutures were given using an absorbable suture material (4-0 Vicryl, Ethicon, Inc, Johnson and Johnson, Somerville, NJ, USA). The sutures were removed 1-week postsurgical.

\section{PROSTHETIC PHASE}

Four months post implant placement, surgical uncovering of the implants is done followed by placement of gingival formers. Two weeks post second stage surgery, open tray impression technique is used to make impression of the mandibular arch (Fig. 8) and closed tray impression technique in the maxillary arch (Fig. 9).

A facebow record is made followed by jaw relation and a wax trial denture. This serves as a guide for the fabrication of a custom poly methyl methacrylate (PMMA) trial framework before milling of the final framework. The PMMA framework is tried in the patient to ensure its passivity (Fig. 10) and then it scanned. The scanned data of the framework and the individual implant platforms are transferred to a design station where the one-piece bar framework is finalized. After design, it is milled from a block of titanium

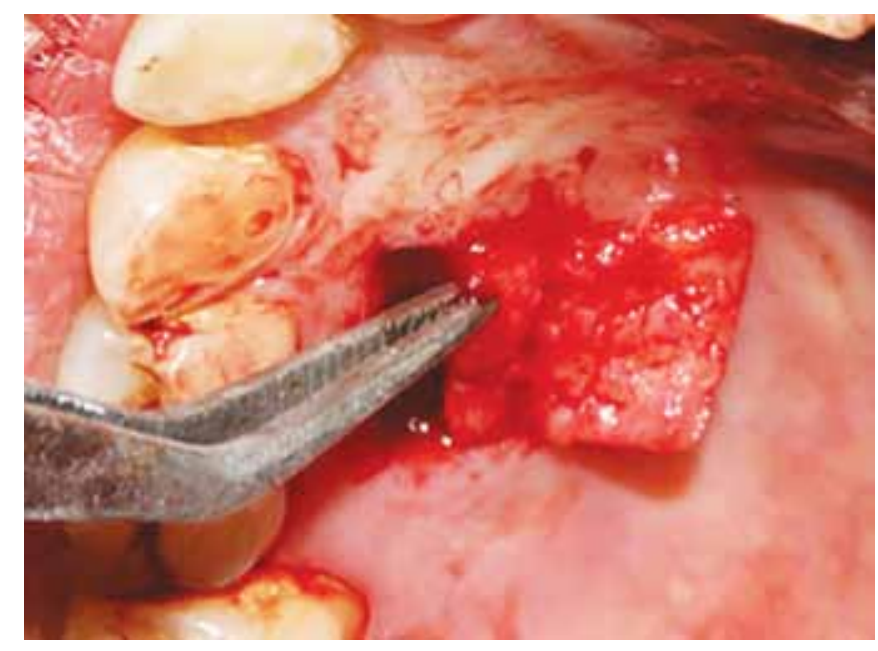

Fig. 6: Connective tissue graft harvested

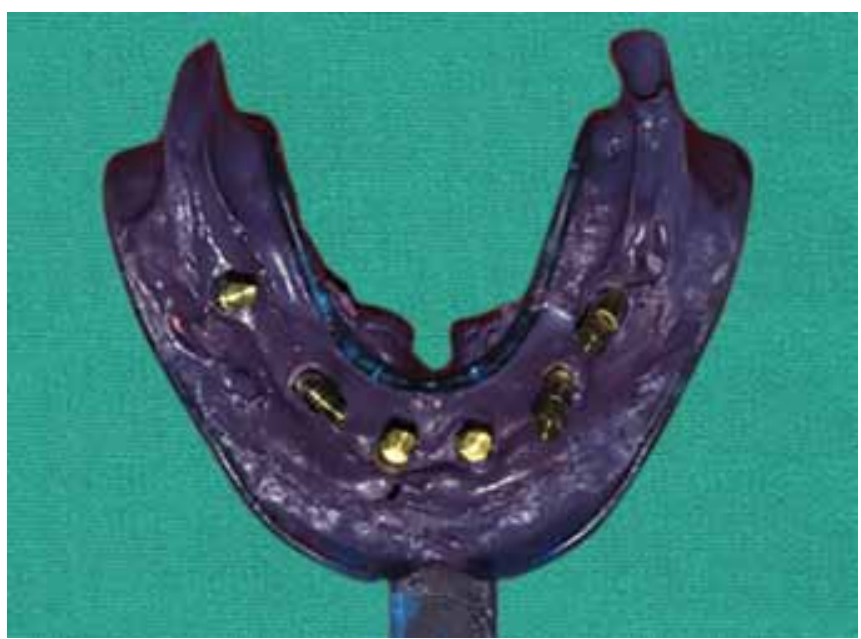

Fig. 8: Open tray impression of the mandibular arch 


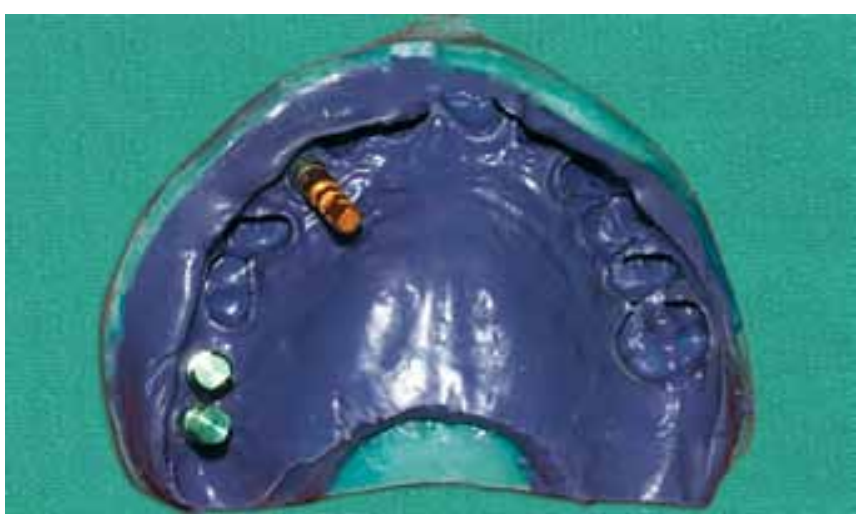

Fig. 9: Closed tray impression of the maxillary arch

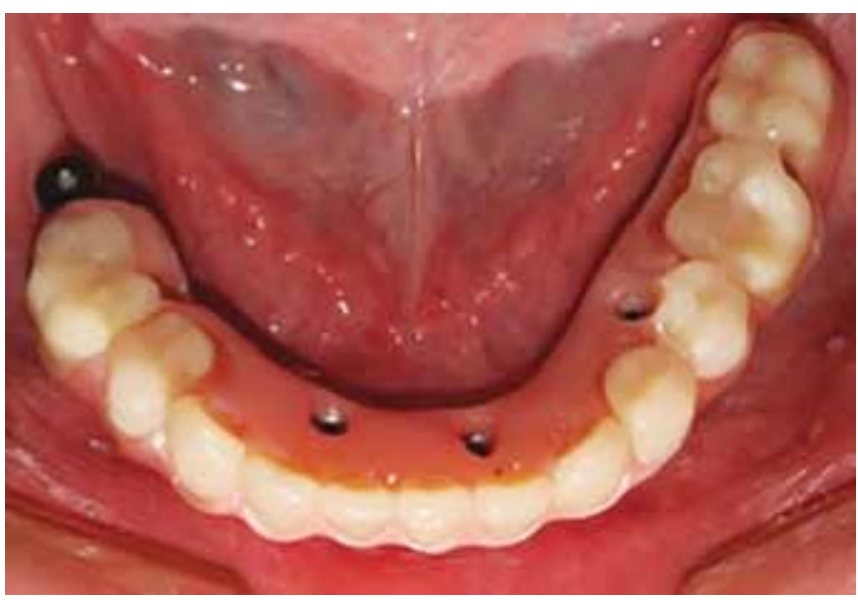

Fig. 11: Wax trial on milled titanium framework

for a precision fit of less than 20 microns. A denture tooth setup is done on this titanium framework and tried in the mouth (Fig. 11). Then, the titanium framework is incorporated into the acrylic processing of the denture.

The hybrid denture extends from the first molar of the right side to the second premolar of the left side. A freestanding fixed prosthesis is fabricated on the implant in the left first molar region. This design of the prosthesis is used to counteract the mandibular flexure, which would occur if the hybrid prosthesis is extended across the mental foramen on both the sides of the mandible. ${ }^{8}$

The coping trial followed by bisque trial is done for the maxillary canine, posterior implants and the mandibular left side posterior implant and bite registration is done in excursive movements followed by programming of the articulator. The presence of natural teeth along with implant prosthesis makes it imperative for the clinician to harmonize the occlusion for longevity of the prosthesis as well as the teeth.

The final prosthesis is screwed in and checked for passivity of its fit (Fig. 12) and the final cementation of the other prosthesis is done and occlusion is reevaluated. Radiographs are made to evaluate the fit of the prosthesis over the implant substructures.

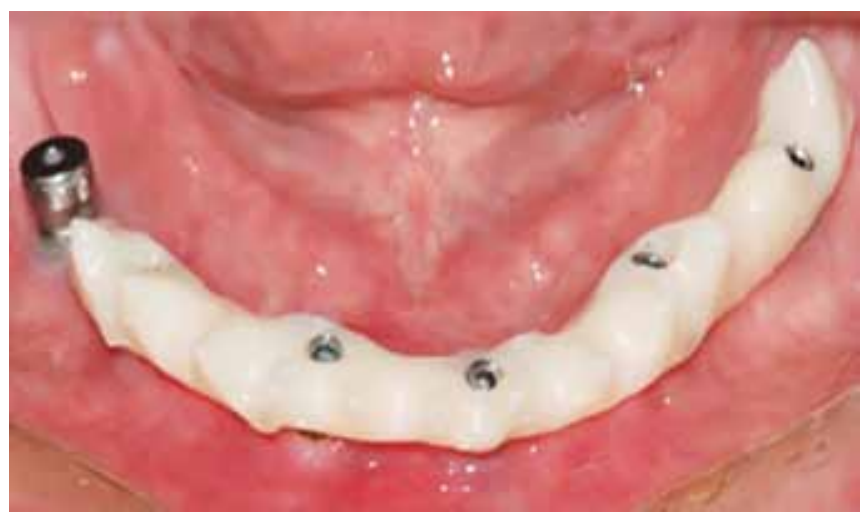

Fig. 10: Poly methyl methacrylate framework trial

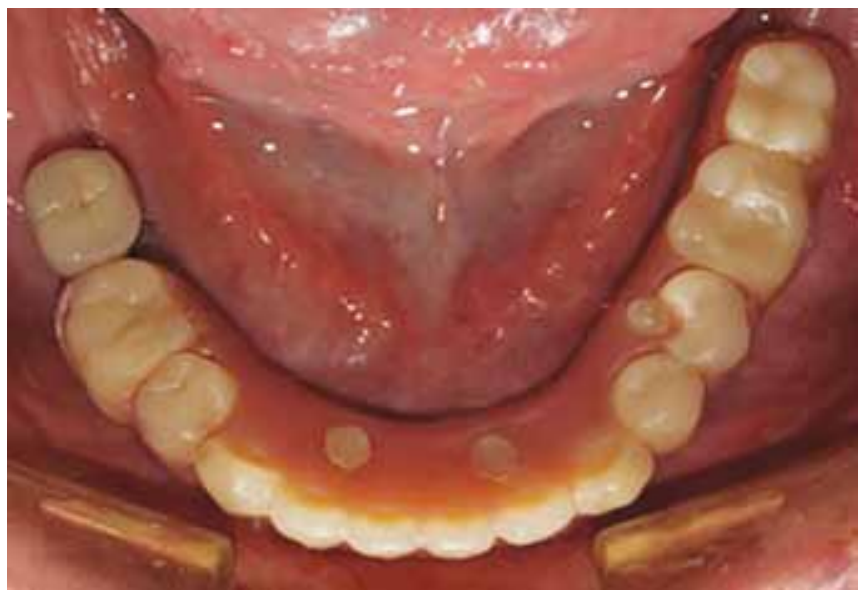

Fig. 12: Final prosthesis inserted

\section{DISCUSSION}

The rehabilitation of edentulous patients with implantsupported hybrid prosthesis has been observed to achieve greater masticatory function and psychological satisfaction than with conventional overdentures. Though, the maintenance of hygiene under the denture is difficult for the patient, the unique screw-retained design of the prosthesis makes it easier for the clinician to maintain it over periodic recalls. Thus, the health of the implants as well as the prosthesis is ensured.

In the case, the treatment plan consists of not extending the hybrid prosthesis over the entire mandible keeping in mind the mandibular flexure. Mc Cartney in 1992 suggested that posterior implants could be subjected to stress-induced microdamage at the bone-implant interface due to mandibular flexure. ${ }^{9}$ Keeping the same principle in consideration, the hybrid prosthesis is not supported by the implant in the molar region.

Producing a passive-fitting substructure for fixed removable screw retained hybrid prosthesis is arguably one of the most technically complex tasks in implant dentistry. In spite of a number of techniques to prevent or correct distortions that occur during impression making, cast pouring, wax- 
ing, casting, indexing, and soldering, errors in the fit of frameworks persist. ${ }^{10}$ A cast titanium framework generally lacked passivity, which led to cumbersome steps of cutting the framework followed by relining and soldering of the framework which made it an arbitrary procedure. Studies found the fit of CAD/CAM-fabricated implant frameworks to be statistically superior to that of the conventional cast frameworks. The passivity is checked in the PMMA trial and corrected after which it is scanned to mill a titanium framework. This prevents the difficulties of relining an illfitting cast framework.

The use of Ostell RFA gives the implantologist ISQ indicating how stable the implant is. It also evaluates the progress of implant osseointegration over different stages. This method of assessing implant stability can provide the clinician with information that can be used to assure their patient that the implant is ready to take prosthetic loading and more importantly, the implant will provide long-term support for a definitive prosthesis. ${ }^{7}$

Implants do not have inserting collagen fiber bundles like a natural tooth; they do have circumferential fibers that sling around the implant like a halo that in essence provides only one compartment, so any inflammation tends to result in a $360^{\circ}$ effect. $^{6}$

There are studies, which state that $80 \%$ peri-implant diseases are a result of the bacterial colonization around the extruded cement. In an implant, which is loaded immediately, the contamination of the surgical site with the extruded cement will impair the osseointegration of the implant. In a 3 -year prospective multicenter study comparing the health of soft tissues surrounding cemented and screw-retained implant restorations, it was reported that the tissues responded more favorably in the latter cases. ${ }^{11,12}$ Hence, a screw-retained temporary was fabricated over the upper left canine.

Thicker soft-tissue biotypes and bone of the labial periimplant tissue have been shown to promote long-term stable gingival margins. Thicker soft tissue biotype also enables the implantologist to provide a better esthetically appealing prosthesis. The use of a connective tissue graft that is harvested from the patient's palate at implant placement helps to achieve a better soft tissue bulk and esthetics. This counteracts the progressive labial gingival recession over the implants.

\section{CONCLUSION}

Every patient has unique treatment needs. Proper diagnosis and treatment plan are important but cannot be all-inclusive. A comprehensive examination, including a thorough medical and dental history, orofacial and dental clinical examination, dental radiographs, impressions, and jaw relation records for mounting casts are important steps leading to a successful oral rehabilitation. Careful integration and sequencing of the different areas of treatment needed, enhances the final result. Dentists must consider the advantages and disadvantages of the available implant prosthetic options and match them to patient's expectations. The mandibular implant-supported hybrid prosthesis and the immediately loaded maxillary implant along with the other free standing implants are recalled and examined over 2 years for occlusion, retention, stability, soft-tissue esthetics and hygiene maintenance. Apart from difficulty in maintaining hygiene around the hybrid prosthesis, all other factors were good on evaluation.

\section{REFERENCES}

1. Chee W, Felton DA, Johnson PF, Sullivan DY. Cemented versus screw-retained implant prostheses: which is better? Int J Oral Maxillofac Implants 1999;14(1):137-141.

2. Taylor TD, Belser U, Meriscske-Stern R. Prosthodontic considerations. Clin Oral Implants Res 2000;11(Suppl 1):101-107.

3. Kapos T, et al. Computer-aided design and computer-assisted manufacturing in prosthetic implant dentistry. Int J Oral Maxillofac Implants 2009;24(Suppl):110-117.

4. Jemt T, Back T, Petersson A. Precision of CNC milled titanium frameworks for implant treatment in edentulous jaw. Int $\mathrm{J}$ Prosthodont 1999;12(3):209-215.

5. Dumbrigue HB, Abanomi AA, Cheng LL. Techniques to minimize excess luting agent in cement retained implant restorations. J Prosthet Dent 2002;87(1):112-114.

6. Rungcharassaeng, Kitichai, et al. Immediate implant placement and provisionalization with and without a connective tissue graft: an analysis of facial gingival tissue thickness. Int J Periodontics Restorat Dent 2012 Dec;32(6):657-663.

7. Peter K May. ISQ: What is it and how to use it in implant dentistry to improve clinical outcomes? Int Dentist-African Edition 2011;1(4):45-49.

8. John A, Hobkirk JA, Havthoulas TK. The influence of mandibular deformation, implant numbers and loading position on detecting forces in abutments supporting fixed implant superstructures. J Prosthet Dent 1998;80(2):169-174.

9. McCartney JW. Cantilever Rests: an alternative to the unsupported distal cantilever of the osseointegrated implant-supported prosthesis for the edentulous mandible. J Prosthet Dent 1992; 68(5):817-819.

10. Naert I, Quirynen M, van Steenberghe D, Darius P. A study of 589 consecutive implants supporting complete fixed prostheses. Part II: Prosthetic aspects. J Prosthet Dent 1992;68(6):949-956.

11. Bauman GR, Mills M, Rapley JW, Hallmon WW. Plaque-induced inflammation around implants. Int J Oral Maxillofac Implants 1992;7(3):330-337.

12. Weber HP, Kim DM, Ng MW, Hwang JW, Fiorellini JP. Periimplant soft tissue health surrounding cement- and screw-retained implant restorations: a multi-center, 3-year prospective study. Clin Oral Implants Res 2006;17(4):375-379. 